OPEN ACCESS

Edited by:

Deyu Xie,

North Carolina State University,

United States

Reviewed by:

Yuru Tong,

Capital Medical University, China

Wangdan Xiong,

Qingdao Agricultural University, China

*Correspondence:

Jinfen Yang

yangj@@zucm.edu.cn

†These authors have contributed equally to this work

Specialty section:

This article was submitted to

Plant Metabolism

and Chemodiversity,

a section of the journal

Frontiers in Plant Science

Received: 15 April 2021

Accepted: 23 July 2021

Published: 12 August 2021

Citation:

Zhao H, Li M, Zhao Y, Lin X, Liang H, Wei J, Wei W, Ma D, Zhou Z and Yang J (2021) A Comparison of

Two Monoterpenoid Synthases

Reveals Molecular Mechanisms

Associated With the Difference of Bioactive Monoterpenoids Between Amomum villosum and Amomum

Iongiligulare.

Front. Plant Sci. 12:695551. doi: 10.3389/fp/s.2021.695551

\section{A Comparison of Two Monoterpenoid Synthases Reveals Molecular Mechanisms Associated With the Difference of Bioactive Monoterpenoids Between Amomum villosum and Amomum longiligulare}

\author{
Haiying Zhao' ${ }^{1+}$, Meng Lit', Yuanyuan Zhao' ${ }^{1}$ Xiaojing Lin' ${ }^{1}$, Huilin Liang ${ }^{1}$, Jieshu Wei², \\ Wuke Wei ${ }^{1}$, Dongming Ma ${ }^{1}$, Zhongyu Zhou ${ }^{3}$ and Jinfen Yang ${ }^{1 *}$ \\ 1 Key Laboratory of Chinese Medicinal Resource from Lingnan (Ministry of Education), Guangzhou University of Chinese \\ Medicine, Guangzhou, China, ${ }^{2}$ School of Pharmacy, Guangzhou Xinhua University, Guangzhou, China, ${ }^{3}$ Key Laboratory \\ of Plant Resources Conservation and Sustainable Utilization, Guangdong Provincial Key Laboratory of Applied Botany, \\ South China Botanical Garden, Chinese Academy of Sciences, Guangzhou, China
}

The fruits of Amomum villosum and Amomum longiligulare are both used medicinally as Fructus Amomi the famous traditional Chinese medicine, however, the medicinal quality of $A$. villosum is better than that of $A$. longiligulare. Volatile terpenoids in the seeds, especially bornyl acetate and borneol, are the medicinal components of Fructus Amomi. The volatile terpenoids and transcriptome of developing seeds of $A$. villosum and A. longiligulare were compared in this study. The result revealed that the bornyl acetate and borneol contents were higher in A. villosum than in A. longiligulare. Additionally, six terpenoid synthase genes (AITPS1-AITPS6) were screened from the transcriptome of $A$. longiligulare, and AITPS2 and AITPS3 were found to share 98 and $83 \%$ identity with AvTPS2 and AvBPPS (bornyl diphosphate synthase) from A. villosum, respectively. BPPS is the key enzyme for the biosynthesis of borneol and bornyl acetate. Biochemical assays improved that AITPS2 had an identical function to AvTPS2 as linalool synthase; however, AITPS3 produced camphene as the major product and bornyl diphosphate (BPP) as the secondary product, whereas AvBPPS produced BPP as its major product. There was only one different amino acid between AITPS3 (A496) and AvBPPS (G495) in their conserved motifs, and the site-directed mutation of A496G in DTE motif of AITPS3 changed the major product from camphene to BPP. Molecular docking suggests that A496G mutation narrows the camphene-binding pocket and decreases the BPPbinding energy, thus increases the product BPP selectivity of enzyme. In addition, the expression level of AvBPPS was significantly higher than that of AITPS3 in seeds, which was consistent with the related-metabolites contents. This study provides insight into the TPS-related molecular bases for the biosynthesis and accumulation differences of the bioactive terpenoids between A. villosum and A. longiligulare. BPPS, the key gene involved in the biosynthesis of the active compound, was identified as a target gene that could be applied for the quality-related identification and breeding of Fructus Amomi.

Keywords: Amomum villosum, Amomum longiligulare, bornyl acetate, transcriptome, bornyl diphosphate synthase, linalool synthase 


\section{INTRODUCTION}

Amomum villosum and Amomum longiligulare are plants of the genus Amomum (family Zingiberacea) that grow in Southeast Asia. According to the China Pharmacopoeia, both the dried and ripe fruits of $A$. villosum and $A$. longiligulare are used as the traditional Chinese medicine, Fructus Amomi (Sharen), which is used to treat digestive diseases such as abdominal pain, vomiting and dysentery (Commission of Chinese Materia Medica, 1999; Commission of Chinese Pharmacopoeia, 2015). In addition, Fructus Amomi is widely used in Chinese cuisine.

The main part of Fructus Amomi with medicinal value is its highly aromatic seeds, which contain rich essential oil. As the most active and abundant ingredient in the essential oil of seeds, bornyl acetate is the important medicinal component, and used as the quality standard of Fructus Amomi (Commission of Chinese Pharmacopoeia, 2015); in addition to its traditional effects (analgesia and antidiarrheic), bornyl acetate has been reported to have antioxidant, anti-inflammatory, antiabortion and anticancer activities (Kim et al., 2013; Chen et al., 2014; Yang et al., 2014; Li and Wang, 2016). However, the content of bornyl acetate in the seeds of A. longiligulare is lower than that of A. villosum (Qin et al., 2017). Because the medicinal quality of $A$. villosum is better than that of $A$. longiligulare, A. villosum is used more widely and has a higher economic value. Genetic identification and evaluation of these two species and other close species with similar morphological traits has been conducted using molecular identification based on DNA barcoding, single nucleotide polymorphisms (SNPs) in the ITS (internal transcribed spacer) and the chloroplast genome (Huang et al., 2014; Cui et al., 2019; Doh et al., 2019). However, metabolomic and transcriptomic comparisons of these species and the molecular mechanisms responsible for the differences in their photochemistry have not yet been investigated.

The constituents of the essential oil in Fructus Amomi from A. villosum were identified, and the most abundant components were found to be monoterpenoids, such as bornyl acetate, borneol and camphor (Yu et al., 2005; De et al., 2011; Xue et al., 2015). In plants, terpenoids are synthesized by the mevalonate (MVA) pathway in the cytoplasm and the 2-C-methyl-D-erythritol-4-phosphate (MEP) pathway in the plastid, while terpenoid synthases (TPS) such as monoTPS and sesqui-TPS catalyze geranyl diphosphate (GPP) and farnesyl diphosphate (FPP) to form diverse terpenoids (Tholl, 2015; Figure 1A). The genes encoding 1-deoxy-D-xylulose5-phosphate reductoisomerase (DXR), 1-deoxy-D-xylulose-5phosphate synthase (DXS) and 3-hydroxy-3-methylglutaryl Coenzyme A reductase (HMGR) for terpenoid backbone biosynthesis in A. villosum have been cloned and identified (Yang et al., 2012; Wang et al., 2014). Additionally, AvBPPS (bornyl diphosphate synthase) and AvPS (pinene synthase), which are involved in monoterpenoid biosynthesis in A. villosum have been cloned and functional characterized (Wang et al., 2018). BPPS catalyzes GPP to produce bornyl diphosphate (BPP), which is the precursor of borneol, and borneol is the precursor of bornyl acetate and camphor (Figure 1B). Therefore, BPPS is the key enzyme for the biosynthesis of borneol, bornyl acetate and camphor, which are the main monoterpenoids of $A$. villosum and $A$. longiligulare. Except for AvBPPS, BPPS genes have been identified from other plants, including Salvia officinalis (SoBPPS), Lavandula angustifolia (LaBPPS), Lippia dulcis (LdBPPS), and Cinnamomum burmanni (CbTPS1) (Wise et al., 1998; Despinasse et al., 2017; Hurd et al., 2017; Ma et al., 2021). However, the enzymes/genes, especially the key enzyme BPPS, involved in the pathway of the main bioactive components in A. longiligulare remain uninvestigated.

As we mentioned above, terpenoids including bornyl acetate, borneol, are the bioactive compounds of $A$. villosum and $A$. longiligulare, knowledge about terpenoids and their biosynthesis of $A$. longiligulare and comparisons of $A$. villosum and $A$. longiligulare can facilitate increased understanding of the biological foundation for differences in their quality. Furthermore, exploring the genes involved in the volatile terpenoid biosynthesis in A. longiligulare and A. villosum will provide insight into the evolution of these two species and lead to improved bioactive terpenoid production.

In recent years, integration of metabolomics and transcriptomics has been utilized to investigate terpenoid biosynthesis in plants (Wei et al., 2016; Chen et al., 2018; Torrens-Spence et al., 2018). Based on our previous report that the $A v B P P S$ gene was expressed at the highest levels in the seeds of 45 days after flowering (DAF) of $A$. villosum (Wang et al., 2018), the 45-DAF seeds of A. longiligulare were used for transcriptomics and metabolomics analysis in this study. The transcriptomics data from A. villosum and A. longiligulare were then used to compare the genes involved in the terpenoids biosynthesis and mine TPS genes. AlTPS2 and AvTPS2 sharing high identity were cloned and characterized. AlTPS3 had high identity with AvBPPS, then was cloned and characterized as well. The comparisons based on the sequence, enzymatic function and expression of these two couples of TPS genes, especially AlTPS 3 and AvBPPS, provided insight into the differences in terpenoids biosynthesis in $A$. villosum and A. longiligulare.

\section{MATERIALS AND METHODS}

\section{Plant Materials}

The flowers and 45-DAF fruits from A. longiligulare and A. villosum were collected and frozen at $-80^{\circ} \mathrm{C}$. The fruit was separated into pericarps and seeds. In addition, the mature fruits (approximately 90-DAF) of A. villosum and A. longiligulare were dried to a constant weight at $50^{\circ} \mathrm{C}$ and then stored in a dryer.

\section{Volatile Terpenoid Extraction and Analysis}

Approximately $0.3 \mathrm{~g}$ of fresh or dry materials was ground frozen in liquid nitrogen, vortexed and then extracted with $1.5 \mathrm{~mL}$ hexane using an ultrasonic cleaner for $0.5 \mathrm{~h}$ followed by incubation at $40^{\circ} \mathrm{C}$ for $1 \mathrm{~h}$. Samples were then centrifuged at $12,000 \mathrm{rpm}$ for $5 \mathrm{~min}$, after which the resulting supernatants were pipetted into new $2 \mathrm{~mL}$ tubes. Next, $1 \mathrm{~mL}$ of hexane extract was 
A
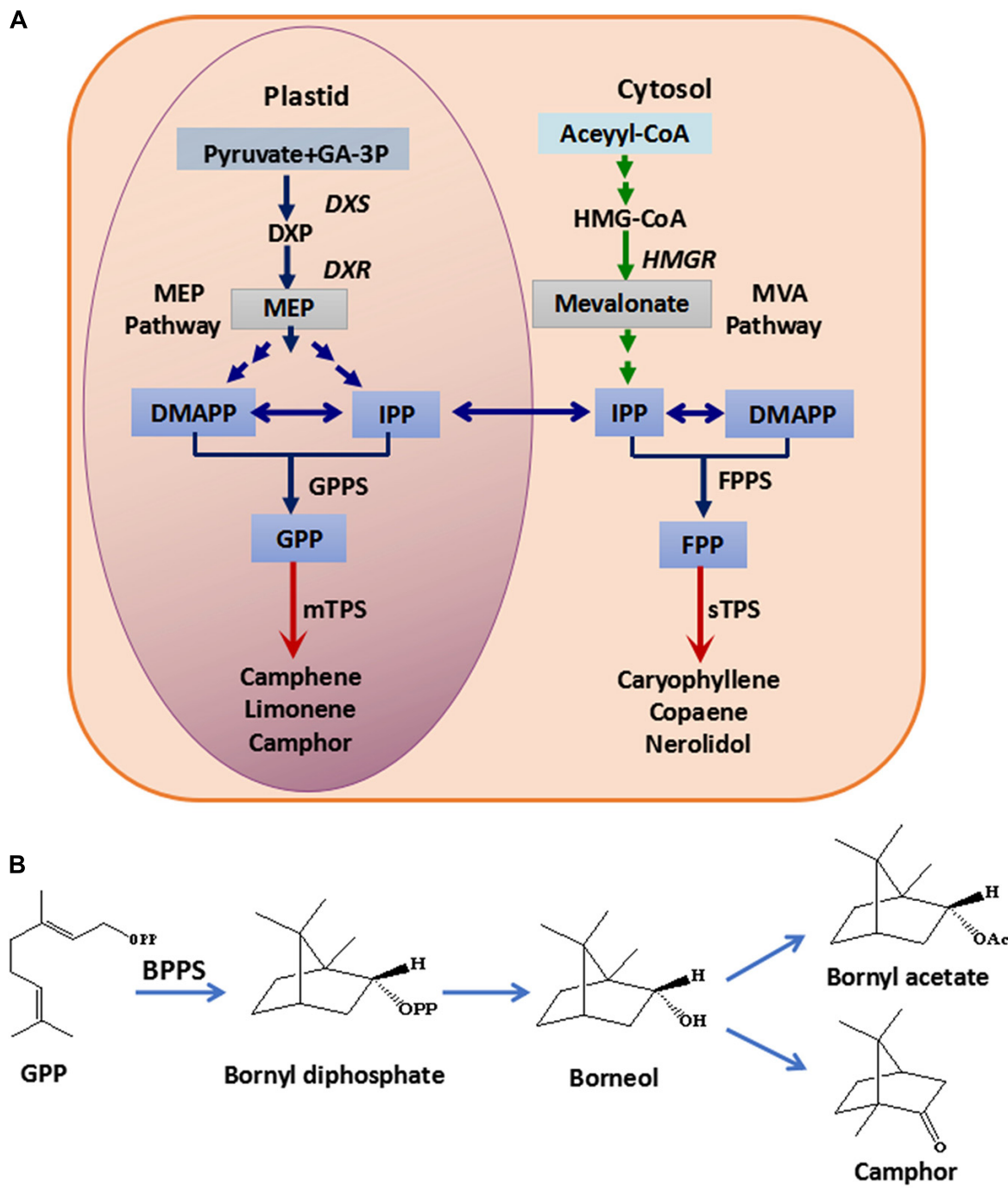

FIGURE 1 | Monoterpenoid and sesquiterpenoid biosynthesis pathway (A) and the biosynthesis pathway from GPP to borneol, camphor and bornyl acetate (B). GA-3P, glyceraldehyde-3-phosphate; HMG-CoA, 3-hydroxy-3-methylglutaryl coenzyme A; DXS, 1-deoxy-D-xylulose-5-phosphate synthase; DXP,

1-deoxy-D-xylulose-5-phosphate; DXR, 1-deoxy-D-xylulose-5-phosphate reductoisomerase; HMGR, 3-hydroxy-3-methyl glutaryl coenzyme A reductase; DMAPP, dimethylallyl diphosphate; IPP, isopentenyl diphosphate; MEP, 2-C-methyl-D-erythritol-4-phosphate; MVA, mevalonic acid; GPPS, geranyl diphosphate synthase; GPP, geranyl diphosphate; FPPS, farnesyl diphosphate synthase; FPP, farnesyl diphosphate; mTPS, monoterpenoid synthase; sTPS, sesquiterpenoid synthase; BPPS, bornyl diphosphate synthase.

pipetted into $1.5 \mathrm{~mL}$ vials for GC-MS analysis. The extracts were then analyzed using an Agilent 7890B Gas Chromatograph with a 5977B inert Mass Selective Detector (Agilent, United States). Helium was used as the carrier gas $(1 \mathrm{~mL} / \mathrm{min})$, then separated on an HP-5MS column $(30 \mathrm{~m} \times 250 \mu \mathrm{m} \times 0.25 \mu \mathrm{m}$ film thickness). For separation, the GC oven temperature was programmed for an initial temperature of $35^{\circ} \mathrm{C}$ for $5 \mathrm{~min}$ followed by an increase of $12^{\circ} \mathrm{C} / \mathrm{min}$ to $300^{\circ} \mathrm{C}$, which was held for $5 \mathrm{~min}$. A NIST $17 /$ demo 1 Mass Spectral Library was used for metabolite identification. The terpenoid compounds were identified by the mass spectral library, after which the main monoterpenoids investigated in this study, including $\alpha$-pinene, camphene, myrcene, limonene, linalool, camphor, borneol and bornyl acetate, were further identified using their authentic standards. The contents were quantified based on bornyl acetate standard curves, and there were three biological replicates for each tissue.

\section{RNA Extraction}

The total RNA of each sample (including seeds, pericarps and flowers) was isolated using an OmniPlant RNA kit (CWbiotech, China), following the manufacturer's protocol. For the RNA extraction of seeds, the following step was added to remove the polysaccharides: after blending by vortexing the lysate and sample, 1/20 volume of isopropyl alcohol and 1/20 volume of high salt solution mixture $(1.2 \mathrm{M} \mathrm{NaCl}+0.8 \mathrm{M}$ sodium citrate) were added to the lysate and blended upside down. The RNA quality was then verified using an ultraviolet spectrophotometer (TIANGEN, China) and checked using RNase free agarose gel 
electrophoresis. RNA with an OD260/OD280 of 1.8-2.2 was used for subsequent analyses.

\section{Transcriptome Sequencing, de novo Assembly and Annotation}

RNA extracted from the 45-DAF seeds was used for the transcriptome sequencing without replicates. The library preparation and transcriptome sequencing, de novo assembly and annotation were conducted by Gene De novo Co., as described previously (He et al., 2018; Wang et al., 2018).

\section{Screening of Candidate AITPS Genes}

The unigenes of candidate AlTPS genes were screened out from the Pfam annotation (Finn et al., 2016) of the transcriptome data of $A$. longiligulare using the keyword "terpene synthase," combining with the local-Blast using the sequences of AvTPS1AvTPS10 (Wang et al., 2018). The unigenes shorter than 500 bp were filtered out.

\section{Cloning of the Full-Length Coding Region of AlTPS2, AvTPS2, and AlTPS3}

The gene-specific primers used for AlTPS2, AvTPS2, and AlTPS3 cloning are listed in Supplementary Table 1. Because AvTPS2 lacks a complete ORF (open reading frame), its $5^{\prime}$-end and $3^{\prime}$-end were amplified using a SMARTer ${ }^{\circledR}$ RACE Kit (Takara, Japan) according to the user manual. The full-length cDNA was amplified using Prime STAR Max DNA Polymerase (Takara, Japan) following the manufacturer's instructions. The PCR conditions used were as follows: $98^{\circ} \mathrm{C}$ for $1 \mathrm{~min}$ followed by 30 cycles of $98^{\circ} \mathrm{C}$ for $10 \mathrm{~s}, 50-60^{\circ} \mathrm{C}$ for $15 \mathrm{~s}$, and $72^{\circ} \mathrm{C}$ for $15 \mathrm{~s}$, and then final extension at $72^{\circ} \mathrm{C}$ for $5 \mathrm{~min}$. The product was then ligated into $\mathrm{pLB}$ cloning vector (Tiangen, China), which was subsequently transformed into Escherichia coli DH5 $\alpha$ and sequenced.

\section{Prokaryotic Expression and Protein Purification of AITPS3, AITPS2, and AvTPS2}

ORFs of AlTPS3, AlTPS2, and AvTPS2 excluding the N-terminal transit peptides were amplified, and proper restriction enzyme sites were added at each end by PCR using the primers described in Supplementary Table 1. The ORFs were then ligated into the pET32a expression vector for AvTPS2 and AlTPS 3 and the pMAL-c5X vector for AlTPS2 using an InFusion Cloning Kit (Takara, Japan). Next, the positive constructs were transformed into competent Rosetta (DE3) cells, and the positive colonies were inoculated into LB media containing $50 \mu \mathrm{g} / \mathrm{mL}$ carbenicillin and $25 \mu \mathrm{g} / \mathrm{mL}$ chloramphenicol and incubated at $37^{\circ} \mathrm{C}$ until the OD 600 reached $0.4-0.6$. The proteins were subsequently induced by incubation in the presence of $0.1 \mathrm{mM}$ isopropyl- $\beta$-D-1-thiogalactopyranoside (IPTG) at $16^{\circ} \mathrm{C}$ for $16 \mathrm{~h}$. Next, the recombined proteins were purified with NINTA resin (Qiagen, Hilden, Germany) or MBP-tag columns (Qiagen, Smart-Life Sciences, China), following the relative manual recommendations: for NI-NTA resin column, unbound proteins were washed with $20 \mathrm{mM}$ imidazole phosphate buffer
(pH 7.4) and then target proteins (AvTPS2 and AlTPS3) were eluted by $200 \mathrm{mM}$ imidazole phosphate buffer; for MBP-tag columns, unbound protein was washed by eluant A $(20 \mathrm{mM}$ Tris$\mathrm{HCl}, 200 \mathrm{mM} \mathrm{NaCl}, 1 \mathrm{mM}$ EDTA and DTT, pH 7.4) and then target protein (AlTPS2) was collected using eluant B (eluant A adding $20 \mathrm{mM}$ maltose). The purified proteins were dialyzed in PD-10 Desalting Columns (GE Healthcare).

\section{Enzyme Assay and Product Analysis}

The enzymatic reaction conditions and methods for the analysis of AlTPS2, AvTPS2, and AlTPS3 were conducted as described by Wang et al. (2018). The enzyme reacted with the substrate GPP for $1 \mathrm{~h}$ at $30^{\circ} \mathrm{C}$, after which samples were dephosphorized by treatment with $1.0 \mu \mathrm{L}$ alkaline phosphatase (Thermo Fisher, United States) for another hour at $37^{\circ} \mathrm{C}$ with $200 \mu \mathrm{L}$ hexane overlaid. The hexane phase was then extracted and used for GC-MS analysis. Enzymatic products were detected based on GC-MS as previously described (Wang et al., 2018). The NIST17 Mass Spectral Library was used for metabolite identification. Additionally, the standards of linalool, camphene, limonene and borneol were utilized for further identification. Each enzyme assay was performed with at least three replicates.

\section{Construction of AITPS3-A496G and AvBPPS-G495A Mutants and Enzyme}

\section{Assay}

The primers for site-directed mutation were listed in Supplementary Table 1. The mutant of AlTPS3-A496G and AvBPPS-G495A were performed using a site-directed mutagenesis kit (TIANGEN, Beijing, China). PCR mixture in a volume of $50 \mu \mathrm{L}$ consisted of $1 \mathrm{U}$ of FastAlteration DNA Polymerase, $5 \times$ FastAlteration Buffer, $10 \mu \mathrm{M}$ primers, and 50 ng of recombinant plasmid pET-32a -AlTPS3. The PCR mixture was denatured at $95^{\circ} \mathrm{C}$ for $2 \mathrm{~min}$, followed by 30 cycles of $20 \mathrm{~s}$ at $94^{\circ} \mathrm{C}, 10 \mathrm{~s}$ at $62^{\circ} \mathrm{C}$, and $2.5 \mathrm{~min}$ at $68^{\circ} \mathrm{C}$, and a final 5 -min extension at $72^{\circ} \mathrm{C}$, the PCR product was incubated at $37^{\circ} \mathrm{C}$ with $D p n I$ restriction enzyme for $1 \mathrm{~h}$ to remove the template plasmid. Then, the PCR product was transformed into competent cells of E. coli, and the mutant site was confirmed by sequencing. The subsequent prokaryotic expression, protein purification were performed following the methods mentioned above. Enzyme assay was performed to compare the products of wild type and mutant using the methods described above with the overnight incubation with GPP instead of $1 \mathrm{~h}$.

\section{Molecular Modeling and Docking}

The secondary structures of the proteins were predicted through SwissModel Workspace, ${ }^{1}$ using 1N24.1.A [crystal structure of (+) -bornyl diphosphate synthase] protein as a template for homology modeling. The reliability of protein models was evaluated by Procheck ${ }^{2}$ and the energy was minimized by SPDBV. The secondary structures of GPP and BPP were downloaded from ZINC. ${ }^{3}$ The molecular docking was performed using AutoDock

\footnotetext{
${ }^{1}$ https://swissmodel.expasy.org/

${ }^{2}$ https://www.ebi.ac.uk/thornton-srv/software/PROCHECK/

${ }^{3}$ http://zinc15.docking.org/
} 
vina with grid points and spacing set as $50 \times 50 \times 50$ and $0.375 \AA$ (Trott and Olson, 2010). The resulting complexes were visualized with PyMOL.

\section{Quantitative Real-Time PCR}

Quantitative real-time PCR (qRT-PCR) was performed to quantify the gene transcriptional expression. The primers (qRTTPS2F/R and qRT-TPS3F/R) used for qRT-PCR are shown in Supplementary Table 1. qRT-PCR was conducted using $2 \times$ M5 HiPer SYBR Premix EsTaq (Mei5 Biotechnology, China) on a CFX 96 Real-Time PCR Detection System (BioRad, United States) following the manufacturers' instructions. The thermal cycling conditions were $95^{\circ} \mathrm{C}$ for $10 \mathrm{~s}$, followed by 40 cycles of $95^{\circ} \mathrm{C}$ for $5 \mathrm{~s}$ and $58^{\circ} \mathrm{C}$ for $30 \mathrm{~s}$. During the reaction, the target gene transcript levels were monitored using a reference gene and calculated using the $2^{-\Delta \Delta \mathrm{Ct}}$ method. All experiments were performed with three biological replicates and three technical replicates.

\section{Bioinformatics Analysis}

Multi-sequence alignment was conducted using the DNAMAN software. A phylogenetic tree was constructed by the neighborjoining method using the MEGA-X software, after which the constructed phylogenetic tree was imported into the iTOL ${ }^{4}$ for modification. Plastid transport peptide was analyzed using the ChloroP 1.1 Server. ${ }^{5}$

\section{RESULTS}

\section{Comparison of Volatile Terpenoids in Seeds of $A$. longiligulare and $A$. villosum}

Seeds are the main medicinal part of Fructus Amomi, which are abundant in volatile terpenoids, therefore, the volatile terpenoids in 45-DAF seeds of A. longiligulare and A. villosum were analyzed by GC-MS. In total, 21 monoterpenoids and 16 sesquiterpenoids were detected in A. longiligulare, while 20 monoterpenoids and 21 sesquiterpenoids were detected in A. villosum, and both the total contents of monoterpenoids and sesquiterpenoids of $A$. villosum are higher than those of $A$. longiligulare (Supplementary Table 2). There were nine monoterpenoids only detected in A. longiligulare or A. villosum, for example linalool was only detected in $A$. longiligulare, while $\gamma$-terpineol was only detected in A. villosum. Monoterpenoids are the majority terpenoids in the seeds, and the percentages of main monoterpenoids, including bornyl acetate, bornel, camphor, D-limonene, myrcene, camphene and $\alpha$-pinene, among the total volatile terpenoids are 50 and $56 \%$ in A. longiligulare and A. villosum, respectively (Supplementary Figures 1A,B). Specifically, the percentage of bornyl acetate of $A$. villosum is higher than that of $A$. longiligulare, however, the percentage of camphor of $A$. longiligulare is higher than that of $A$. villosum (Supplementary Figures 1C,D). Additionally, considering the mature and dried fruits are used as traditional medicine, the main monoterpenoids of mature and

${ }^{4}$ https://itol.embl.de/

${ }^{5}$ http://www.cbs.dtu.dk/services/ChloroP/ dried seeds of $A$. longiligulare and A. villosum were analyzed as well, and compared with the data of developing seeds. In mature seeds, bornyl acetate and camphor are the most abundant monoterpenoid in A. villosum and A. longiligulare, respectively (Supplementary Table 3 ). The contents of bornyl acetate and borneol of $A$. villosum are higher than those of $A$. longiligulare, but the content of camphor of $A$. longiligulare is higher than that of A. villosum. Camphor, borneol and bornyl acetate are produced from the same precursor BPP (Figure 2B); the total contents of these BPP-related terpenoids are higher in A. villosum than in A. longiligulare in spite of 45 -DAF seeds or mature seeds (Table 1).

\section{Transcriptomics Analysis and Comparison of Genes Involved in Terpenoid Backbone Biosynthesis Between $A$. longiligulare and $A$. villosum}

In order to mine the BPPS gene and to explore the other genes involved in the terpenoid biosynthesis of A. longiligulare, the 45-DAF seeds were used for transcriptome sequencing. Highthroughput sequencing and de novo assembly yielded 62,433 unigenes, and 36,746 unigenes (58.86\%) were totally annotated using the major public databases Nr, KOG, Swiss-Prot and KEGG (Supplementary Table 4 and Supplementary Figure 2). The transcriptome data were submitted to the NCBI and assigned the SRA accession number SRR10769494. Based on the KEGG annotation, 1,527 unigenes were mapped to the biosynthesis of secondary metabolites, and 88 unigenes were annotated to terpenoid backbone biosynthesis. The KEGG annotation data of the 45-DAF seeds transcriptome of A. villosum (Wang et al., 2018) were used to compare the genes involved in the upstream pathway of terpenoid biosynthesis with A. longiligulare. The results revealed that all genes involved in the MVA and MEP pathway were annotated in both A. longiligulare and A. villosum transcriptome data, but more unigenes were annotated in total in A. villosum (62) than in A. longiligulare (58) (Supplementary Table 5). The identities of relative genes of $A$. villosum and A. longiligulare were all higher than $80 \%$.

\section{Six AITPS Candidate Genes Were Screened Out From the Transcriptome, and AITPS3, AITPS2, and AvTPS2 Were Cloned}

Based on the Pfam annotation of the transcriptome data of A. longiligulare and the local-Blast result via AvTPSs (Wang et al., 2018), six AlTPS unigenes with longer length were screened out (Supplementary Table 6). Of these genes, three (AlTPS1AlTPS3) were predicted to encode the monoterpenoid synthase gene, and AlTPS3 presented the highest RPKM expression value. According to the nucleotide sequence alignment, AlTPS3 had the highest (95\%) identity with AvBPPS, which has been identified as bornyl diphosphate synthase from A. villosum (Wang et al., 2018). AlTPS2 shared 99\% identity with AvTPS2, which had been previously screened from the transcriptome of A. villosum but yet cloned (Wang et al., 2018). AlTPS2, AvTPS2 
A

RRX $\mathbf{W}$

AvTPS2

AlTPS

A ITPS2

AvTPS2

AvBPPS

A ITPS2

AvTPS2

AlTPS3
AvBPPS

AITPS2

AvTPS2

AITPS3

AvBPPS

AITPS2

AvTPS2

AITPS3

AvBPPS

AITPS2

AvTPS2

AITPS3

AvBPPS

AITPS2

AvTPS2

AITPS3

AvBPPS

MSLFLAPP. SYFPLESLRRSTAANOP

RRSSPYQPN

RXR IEAGNGADDADN.

\section{DDXXD}

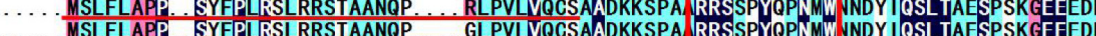
MATRO TYS LSPP

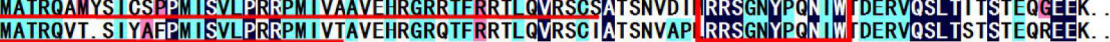

\section{TTKRLMLLKERISEVDCEKKEVEEQLRLIDHLQQLGVAYHFKDDDKGSLRNFHASLEE ISSTFKEDLHATALLFRLLRENGFSITED 162 RERRNVLKEOTRNI RERRNVLKEOTRNLDLEOOOVAEOLREIDHLOLGVAYIFKDEDSDVLSRLHASLDGVSOLEDDLHALALLFRLLPA SGESVSOD}
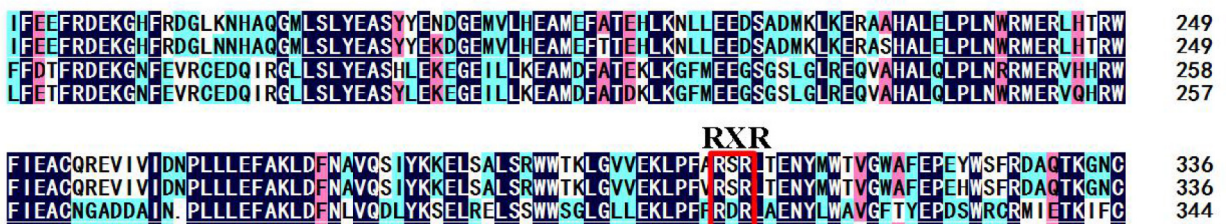

作

FVTMIDDYYD VYGNLDELELFISVVDRWDINAIDQLPDYMKILFLALFNTINDDGYKVTKEKGLDIIPYLKRSWADLCKAYLVEAKW

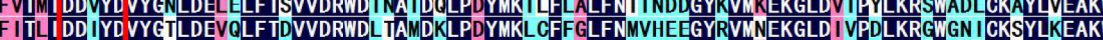

LVIMIDD IYY VYGTLDELOLF JDVVDRWDL TAMDKLPEYMKLCEFALFNMVHEEGRVMKEKGLDIVPDLKR IWGNOCKSYLKEAKW

\section{NSE/DTE}

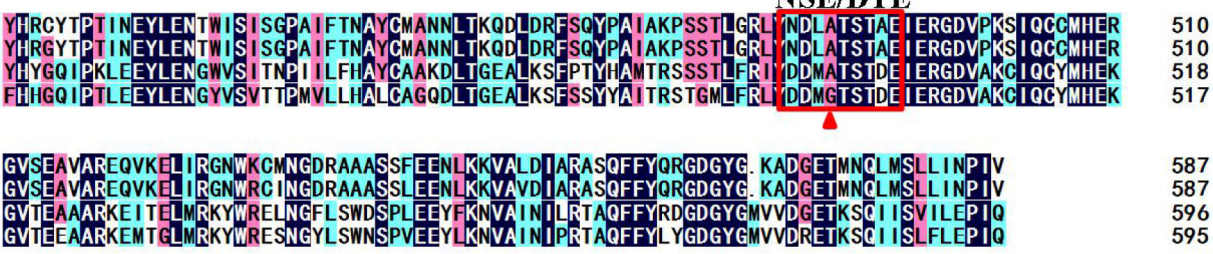

B

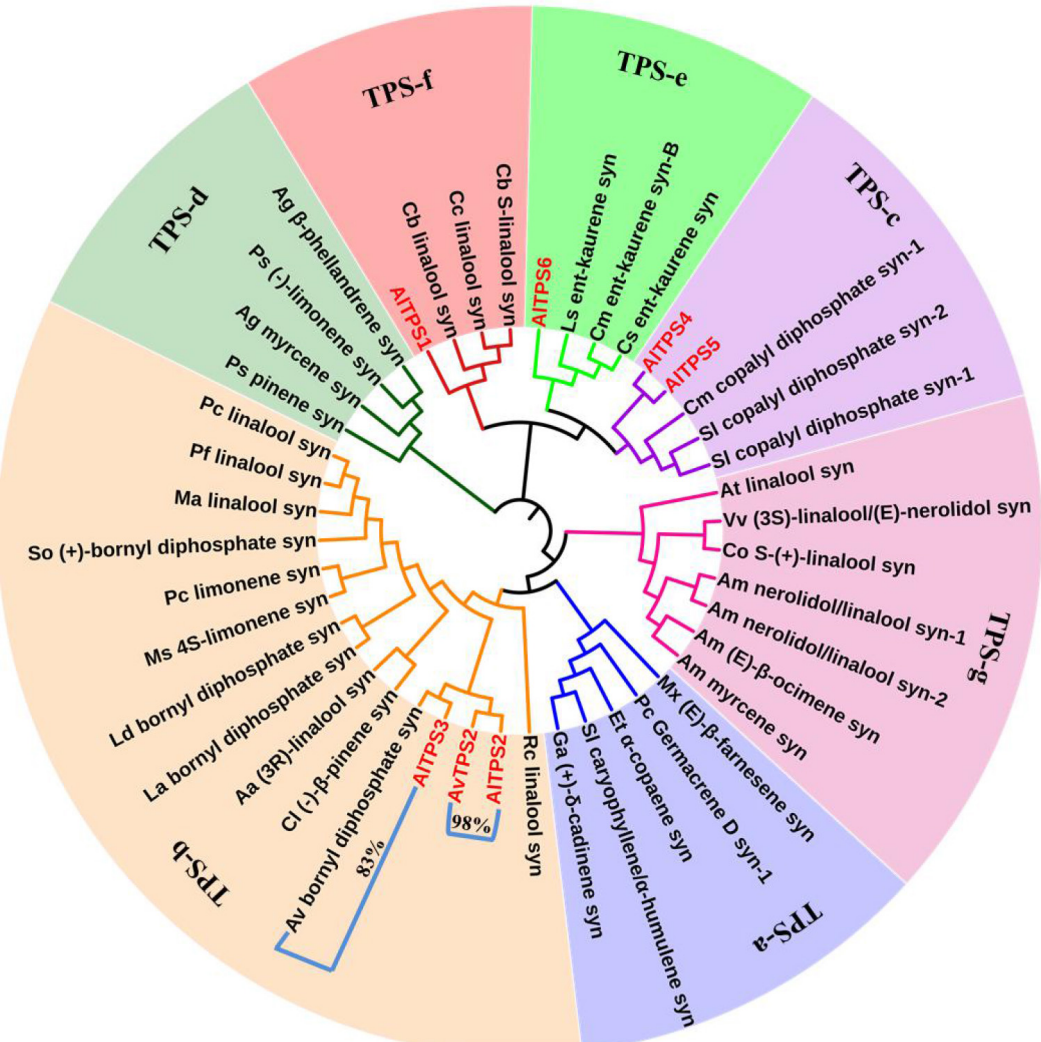

FIGURE 2 | Amino acid sequence alignment of AITPS2, AITPS3, AVTPS2 and AVBPPS (A) and phylogenetic analysis of AITPSs from A. longiligulare, AvTPS2 and terpenoid synthases from other plants (B). The red underline indicates the plastid transport peptide. The red box represents the conserved motifs. The red triangle indicates the different amino acids between AITPS3 and AvBPPS. The number in (B) represents the identity of the amino acid sequences of two genes clustered into one branch. 
TABLE 1 | Comparison of main monoterpenoid contents $(\mu \mathrm{g} / \mathrm{mg})$ in seeds of $A$. logiligulare and A. villosum.

\begin{tabular}{|c|c|c|c|c|c|}
\hline \multirow[t]{2}{*}{ Compound } & \multirow[t]{2}{*}{ Formula } & \multicolumn{2}{|c|}{ 45-DAF seeds (fresh) } & \multicolumn{2}{|c|}{ Mature seeds (dry) } \\
\hline & & A. longiligular & A. villosum & A. longiligular & A. villosum \\
\hline$\alpha$-Pinene & $\mathrm{C}_{10} \mathrm{H}_{16}$ & $0.67 \pm 0.04$ & $1.75 \pm 0.04$ & $0.88 \pm 0.08$ & $1.63 \pm 0.19$ \\
\hline Camphene & $\mathrm{C}_{10} \mathrm{H}_{16}$ & $1.73 \pm 0.23$ & $3.13 \pm 0.06$ & $4.31 \pm 0.58$ & $4.85 \pm 0.61$ \\
\hline Myrcene & $\mathrm{C}_{10} \mathrm{H}_{16}$ & $1.33 \pm 0.16$ & $1.21 \pm 0.02$ & $0.73 \pm 0.07$ & $2.04 \pm 0.26$ \\
\hline D-Limonene & $\mathrm{C}_{10} \mathrm{H}_{16}$ & $1.81 \pm 0.22$ & $2.84 \pm 0.04$ & $2.03 \pm 0.21$ & $3.74 \pm 0.49$ \\
\hline Camphor & $\mathrm{C}_{10} \mathrm{H}_{16} \mathrm{O}$ & $2.45 \pm 0.28$ & $1.35 \pm 0.03$ & $16.25 \pm 1.20$ & $8.70 \pm 0.24$ \\
\hline Borneol & $\mathrm{C}_{10} \mathrm{H}_{18} \mathrm{O}$ & $0.85 \pm 0.08$ & $1.25 \pm 0.03$ & $1.83 \pm 1.36$ & $3.04 \pm 0.38$ \\
\hline Bornyl acetate & $\mathrm{C}_{12} \mathrm{H}_{20} \mathrm{O}_{2}$ & $5.23 \pm 0.54$ & $9.52 \pm 0.11$ & $7.47 \pm 0.86$ & $16.52 \pm 1.00$ \\
\hline BPP-related terpenoids* & - & $8.53 \pm 0.90$ & $12.13 \pm 0.61$ & $25.55 \pm 2.42$ & $28.25 \pm 1.61$ \\
\hline
\end{tabular}

*BPP-related terpenoisd means the terpenoids produced from BPP (bornyl diphosphate), including camphor, borneol and bornyl acetate.

and AlTPS3 were cloned in this work. The complete ORFs of both AlTPS 2 and AvTPS2 were 1,764 bp, encoding 587 amino acids with a predicted 34 -amino-acid plastid transit peptide on the N-terminal (Figure 2A). The complete ORF of AlTPS3 was 1,791 bp, and it encoded 597 amino acids with a predicted 47amino-acid transit peptide on its N-terminal, however, AvBPPS encoded 596 amino acids and its predicted transit peptide was shorter than AlTPS3 (Figure 2A). The gene and deduced amino acid sequences of AlTPS2, AvTPS2, and AlTPS3 have been submitted to GenBank under the accession numbers MN829548, MN829551, and MN829549, respectively.

The deduced amino acid sequences of six candidate AlTPSs and AvTPS2 (Supplementary Table 7), and other functional TPS identified from subfamilies a-g (Supplementary Table 8) were used to construct phylogenetic trees (Figure 2B). AlTPS2, AvTPS2, and AlTPS3 were clustered into the TPS-b subfamily, which is composed of monoterpenoid synthases. AlTPS1 was clustered into the TPS-f subfamily, while AlTPS4 and AlTPS5 were clustered into the TPS-c subfamily, and AlTPS6 was clustered into the TPS-e subfamily. The terpenoid synthases in the TPS-c and TPS-e subfamilies were found to be diterpenoid synthases. Moreover, AlTPS2 and AvTPS2 were clustered into one branch, while AlTPS3 and AvBPPS were clustered into another branch. Alignment of AlTPS2, AvTPS2, AlTPS3, and AvBPPS showed that they all contained the conserved monoTPS motif, RRX 8 W, RXR, DDXXD, and NSE/DTE (Figure 2A). Comparison of the amino acid sequences revealed that the identity of AlTPS2 and AvTPS 2 was $98 \%$. The four conserved motifs of AlTPS2 and AvTPS2 were completely consistent, and their other amino acid regions were highly conserved as well (Supplementary Figure 3). However, the amino acid identity of AlTPS3 and AvBPPS was only 83\%, although they were clustered into a close branch (Figure 2B). Therefore, we speculated that the functions of AvTPS2 and AlTPS2 might be the same, while the function of AlTPS3 might be very similar to AvBPPS.

\section{AITPS2 and AvTPS2 Were Characterized as Linalool Synthase}

The transit peptide of monoterpenoid synthases often reduces soluble protein expression; therefore, the transit peptides of AlTPS2 and AvTPS2 were truncated. The remaining coding regions of AlTPS2 and AvTPS2 were then sub-cloned into expression vectors. The recombinant proteins of AlTPS2 and AvTPS2 were induced and purified using MBP-tag columns and NI-NTA resin, respectively. SDS-PAGE showed that the recombinant proteins of AlTPS2 (fused with MBP-tagged) and AvTPS2 (fused with His-tagged) were about 108 and $89.0 \mathrm{kDa}$, respectively, as predicted (Supplementary Figure 4). To verify the function of AlTPS2 and AvTPS2, the AlTPS2 and AvTPS2 proteins were incubated with GPP or FPP, after which the reaction products were detected using GC-MS. When the substrate was GPP, AlTPS2, and AvTPS2 produced the monoterpene product linalool (Figure 3A) based on the comparison of retention time and mass spectra with those of the linalool standard (Figure 3B). AlTPS2 and AvTPS2 could not catalyze FPP to form products (data not shown). The optimum pH of AlTPS2 and AvTPS2 was $\mathrm{pH} 8$ and $\mathrm{pH}$ 5, respectively (Supplementary Figures 5A,C), and both showed much higher activity in the presence of $\mathrm{Mg}^{2+}$ than $\mathrm{Mn}^{2+}$ (Supplementary Figures 5B,D).

\section{Correlation of Gene Expression Levels of AITPS2 and AvTPS2 With Linalool \\ Accumulation}

To investigate the correlation of the expression levels of AvTPS2 and AlTPS 2 with metabolite accumulation, qRT-PCR of AvTPS2 and AlTPS2 was performed. As the organ accumulating linalool, flowers were also used for this analysis besides pericarps and seeds. Both AlTPS2 and AvTPS2 were expressed in flowers and pericarps higher than in seeds (Figure 4A). The expression level of AlTPS2 in the flowers of A. longiligulare was much higher than that of AvTPS2 in the flowers of A. villosum, however, the linalool content in the flowers of A. villosum was much higher than that of $A$. longiligulare, and so as the linalool content in the pericarps of A. villosum (Figure 4B). The AlTPS2 or AvTPS2 expression in different tissues was not consistent with the linalool accumulation, therefore we speculate that in addition to AvTPS2 or AlTPS2 there are other terpenoid synthases responsible for linalool biosynthesis in A. villosum or A. longiligulare.

\section{AITPS3 Produced Camphene and Bornyl Diphosphate as the Top Two Products}

The transit peptide of AlTPS3 was truncated, and the remaining coding region was sub-cloned into the $\mathrm{pET} 32 \mathrm{a}$ expression vector. 


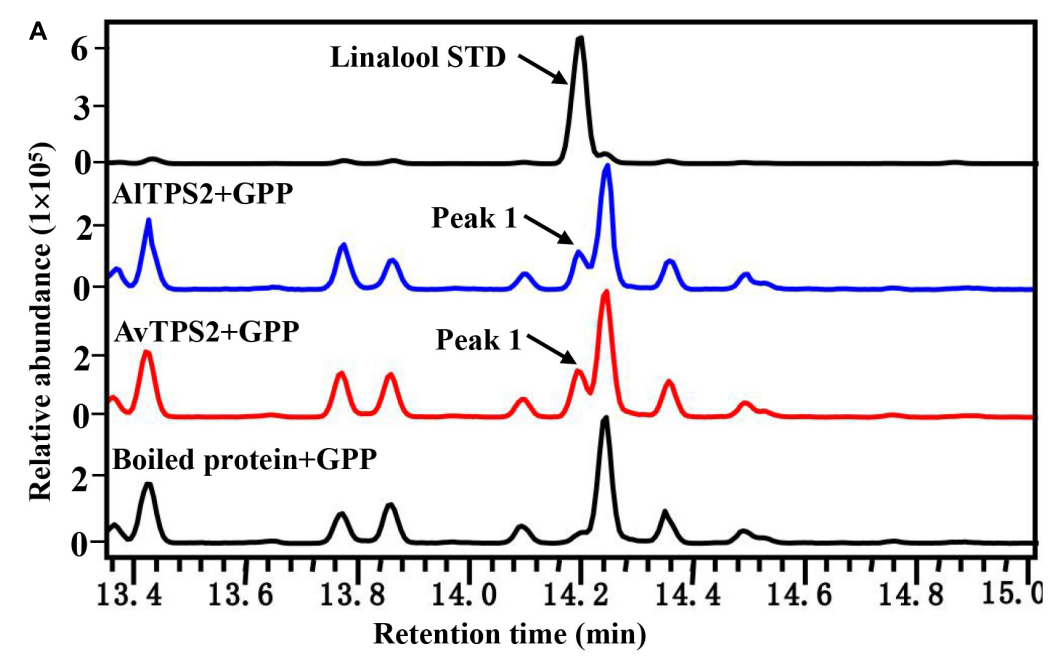

B
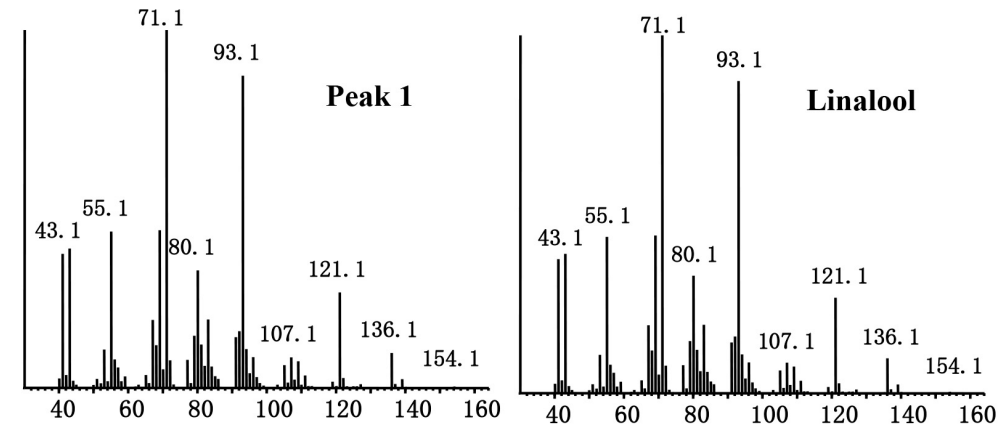

FIGURE 3 | Analysis of reaction products generated by recombinant protein AITPS2 and AvTPS2 from GPP. (A) The GC-MS chromatogram of the monoterpenoid products generated by AITPS2 and AvTPS2 protein and the linalool standard. (B) Mass spectra of peak 1 in the NIST17 library compared with the mass spectra of the linalool standard.
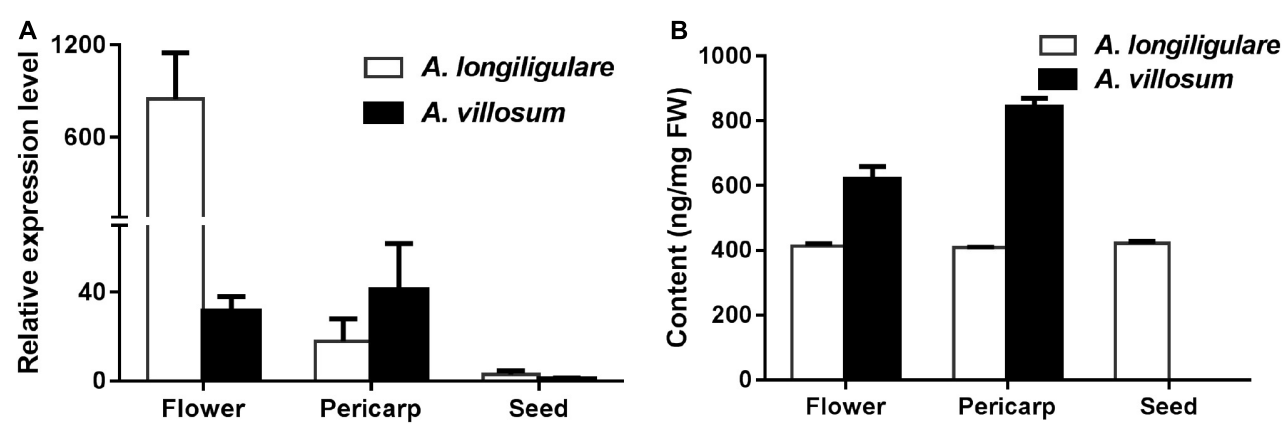

FIGURE 4 | Expression levels of AITPS2 and AvTPS2 and content of linalool in different tissues of A. longiligulare and A. villosum. (A) The expression levels of AITPS2 and AvTPS2. (B) Content of linalool.

The AlTPS3 recombinant protein was approximately $69.36 \mathrm{kDa}$ (Supplementary Figures 4C,D). GC-MS analysis of the in vitro enzymatic assay revealed that the AlTPS3 recombinant protein catalyzed GPP to produce camphene as the major product and limonene as the main by-product, which is similar to AvBPPS (Supplementary Figure 6). In addition, minor amounts of tricyclene, $\alpha$-pinene, $\beta$-terpineol, and terpinolene were detected in the AlTPS3 enzymatic product. After dephosphorization, borneol, the dephosphorylated product of borneol diphosphate (BPP), was detected in both AlTPS3 and AvBPPS enzymatic products, as well as limonene and camphene (Figure $\mathbf{5 A}$ and Supplementary Figure 7). Other trace by-products, such as $\beta$-myrcene and tricyclene, were also detected but not marked in Figure 5A.

Comparison of the product percentages of AlTPS3 and AvBPPS revealed that camphene was the major product (62.5\%) 

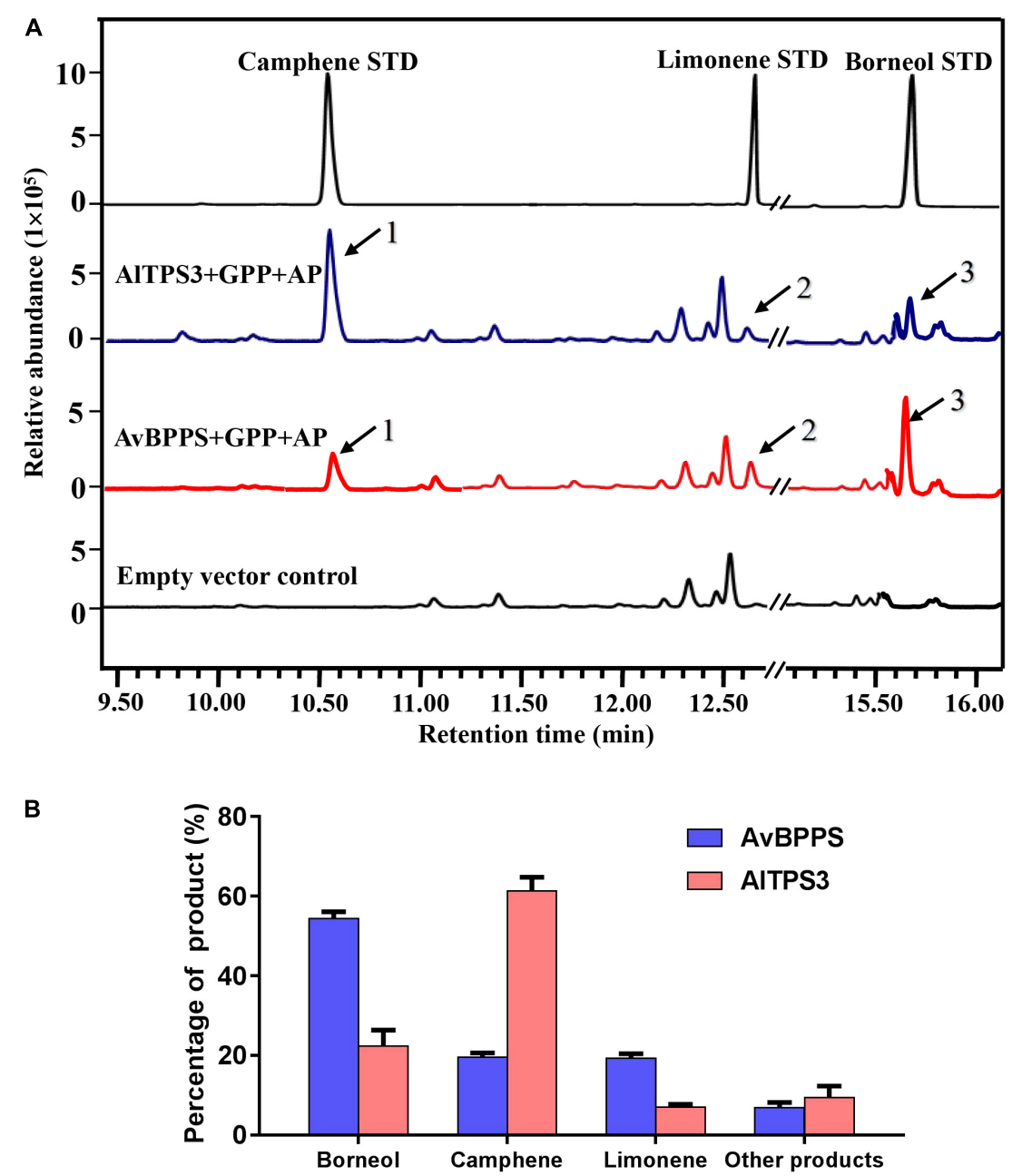

FIGURE 5 | Analysis of reaction products generated by recombinant protein AITPS3 and AvBPPS from GPP. (A) The GC-MS chromatogram of the monoterpenoid products generated by AITPS3 and AvBPPS protein and the camphene, limonene and borneol mixed standards. Mass spectra of peak 1 , peak 2 and peak 3 in the NIST17 library compared with mass spectra of the camphene, limonene and borneol standards shown in Supplementary Figure 6. (B) Product percentage of AvBPPS and AITPS3.

of AlTPS3, while borneol was the second most abundant product $(22.1 \%)$ (Figure 5B); however, the major product of AvBPPS was borneol (57.6\%), while minor products consisted of camphene $(21.0 \%)$, limonene and $\beta$-myrcene (Figure 5B). AlTPS3 produced an approximately $3: 1$ ratio of camphene and borneol, however, AvBPPS produced the reverse ratio of camphene and borneol (Supplementary Figure 8). The optimum $\mathrm{pH}$ of AlTPS3 and AvBPPS was $\mathrm{pH} 7$ and $\mathrm{pH}$ 6, respectively (Supplementary Figures 9A,C). The activity of AlTPS3 showed higher dependence of $\mathrm{Mg}^{2+}$ than $\mathrm{Mn}^{2+}$ (Supplementary Figures 9B,D).

\section{The Site-Directed Mutation of A496G in DTE Motif of AITPS3 Changed the Major Product From Camphene to BPP}

According to the sequence alignment of AlTPS3 and AvBPPS, the sequences of other three conserved motifs were complete identical between AlTPS3 and AvBPPS, there was only one different amino acid, A496 for AlTPS3 and G495 for AvBPPS, in the DTE motif (Figure 3A). In addition, according to the sequence alignment of AlTPS3 and other known BPPSs, including AvBPPS, SoBPPS, LaBPPS, and LdBPPS, the glycine in DTE motif of BPPSs with BPP as main product is highly conserved only the amino acid of AlTPS3 in this site is alanine (Figure 6A). To identify the function of this different amino acid residue, the site-directed mutant of AlTPS3-A496G and AvBPPSG495A were constructed, and the overnight reaction with GPP was performed. The comparison of mutant enzyme with AlTPS wild-type enzyme revealed that site-directed mutation of A496G in the conserved DTE motif changed the major product from camphene to BPP (borneol as final product), and no other product was detected expect for camphene (41\%) and borneol (59\%) (Figure 6B). Compared with AvBPPS wildtype, AvBPPS-G495A increased the percentage of camphene and decreased the percentage of BPP (borneol), which is the 
opposite result of AvTPS3-A496G, although BPP is still the main product of AvBPPS-G495A (Supplementary Table 9). The results suggest that the glycine in DTE motif affects product BPP selectivity of enzyme.

In order to explain how A496G affects the product selectivity of AvTPS3, molecular docking was conducted based on the virtual three-dimensional structures of AlTPS3-WT and AlTPS3A496G, which were predicted by homology modeling using SoBPPS crystal structure (Whittington et al., 2002). The evaluation results of Procheck show that the protein model is reliable (Supplementary Figure 10). Superimposition of AlTPS3A496G on AlTPS3-WT in their catalytic pockets reveals that their substrate-binding and BPP-binding pockets are similar, although AlTPS3-A496G has a few more active residues than AlTPS3-WT (Y425 and S450 for the GPP-binding pocket and D494 for BPP-binding pocket) (Figures 7A,B). However, the camphene-binding pocket of AlTPS3-WT (colored in white) is obviously bigger than that of AlTPS3-A496G (colored in blue), and AlTPS3-WT has four more active residues than AlTPS3A496G (Figure 7C). Compared with AlTPS3-WT, the smaller camphene-binding pocket of AlTPS3-A496G may hinder the production of camphene. Additionally, the binding energy of AlTPS3-A496G with BPP is lower than that of AlTPS3-WT ( -6.0 vs. $-4.7 \mathrm{Kcal} / \mathrm{mol}$ ) (Supplementary Table 10), which may facilitate the BPP production. Taken together, we speculate that A496G mutation narrows the camphene-binding pocket and decreases the BPP-binding energy, thus increases the BPP selectivity of products.

\section{The Expression Level of AvBPPS Was Higher Than That of AITPS3 in Seeds and Matched the Related Terpenoids Accumulation}

To compare the expression of AlTPS3 and AvBPPS, 45-DAF pericarps and seeds of $A$. villosum and $A$. longiligulare were used to perform qRT-PCR. The results revealed that both AvBPPS and AlTPS3 were highly expressed in seeds, but hardly expressed in pericarps (Figure 8A). The expression level of AvBPPS in seeds of A. villosum was nearly three times of that of AlTPS3 in seeds of A. longiligulare. The high expression level of AvBPPS was correlated with the high content of corresponding terpenoids, including BPP-related terpenoids (borneol, camphor and bornyl acetate) and camphene in seeds, and the expression level of AlTPS3 matched the contents of corresponding monoterpenoids as well (Figures 8B,C).

\section{DISCUSSION}

According to the China Pharmacopoeia, the fruits from both A. villosum and A. longiligulare are the origin of Fructus Amomi; however, the therapeutic effect of $A$. villosum is better than A. longiligulare. Terpenoids are the bioactive components of many medicinal plants. The difference of volatile terpenoids between $A$. villosum and A. longiligulare has yet been reported. In this study, monoterpenoids were detected as the dominant volatile terpenoids in the seeds of A. longiligulare, as well as in A. villosum. Seven main monoterpenoids in 45-DAF and mature seeds, including bornyl acetate, bornel and camphor were further compared between these two species. The contents of borneol and bornyl acetate, the main bioactive compounds, were higher in the seeds of $A$. villosum than in A. longiligulare, in addition, camphor was the most abundant monoterpenoid but not bornyl acetate in A. longiligulare. These results suggest the phytochemical basis for the better medicinal quality of $A$. villosum than A. longiligulare.

Six candidate terpenoid synthases genes (AlTPS1-AlTPS6), including three monoterpenoid synthase genes, were mined from the transcriptome data of A. longiligulare. Since only one tissue, 45-DAF seeds, was used for transcriptome sequencing in this study to identify the BPPS gene, these six AlTPS genes might only be part of the TPS family of A. longiligulare. To understand the AlTPS family more comprehensively, transcriptome sequencing with more tissues, including fruits of different developing stages, should be performed in the future.

AlTPS2 and AvTPS2 were characterized as linalool synthase; they have the same enzymatic function consistent with their high identity in sequence. Linalool is a major volatile compound widely distributed in plant, especially in flowering plants. This compound is frequently used as an ingredient of perfumes, food and household detergents (Mei et al., 2015). Linalool is also an important intermediate in the synthesis of vitamin $\mathrm{E}$ during industrial production (Aprotosoaie et al., 2014). LINS, the TPS that synthesizes linalool, has been identified in many plants, such as RcLINS from rose and LaLINS from lavender (Landmann et al., 2007; Magnard et al., 2018). AlTPS2 and AvTPS2 were expressed with the highest levels in flowers correlating with the accumulation of linalool. Because the expression levels of AvTPS2 or AlTPS2 did not match the linalool contents in flowers, pericarps and seeds of A. villosum and A. longiligulare, it is speculated that there might be more than one LINS gene responsible for linalool synthesis in A. villosum or A. longiligulare.

Bornyl acetate, which was found to be the most abundant volatile terpenoid in $A$. villosum and the second most abundant volatile terpenoid in A. longiligulare (Table 1), is the quality standard and the most active ingredient of Fructus Amomi. BPPS is a special TPS that catalyzes GPP to form BPP, which is an unusual terpenoid intermediate containing a pyrophosphate group (Christianson, 2017). BPP is subsequently converted to borneol by dephosphorylation, after which the borneol is converted to bornyl acetate by acetyl transferation (Adam and Croteau, 1998). Therefore, BPPS is the key enzyme responsible for the biosynthesis of bornyl acetate. In this study, an enzyme producing BPP has been discovered from A. longiligulare. All these BPPSs, including AvBPPS, SoBPPS, LaBPPS, and LdBPPS, can produce BPP, camphene and limonene as products; however, unlike the other four BPPSs that produce BPP as the major product, AlTPS3 produces camphene as the major product and $\mathrm{BPP}$ as the secondary product with the ratio of camphene to BPP 3:1. All of the conserved motifs of AlTPS3 and AvBPPS were consistent, except for one amino acid site, A496 for AlTPS3 vs. G495 for AvBPPS in the DTE motif, furthermore, only this site of AlTPS3 is alanine, while this site of other BPPSs which produce 
A AlTPS3
AvBPPS
LaBPPS
SoBPPS
LdBPPS
DTE

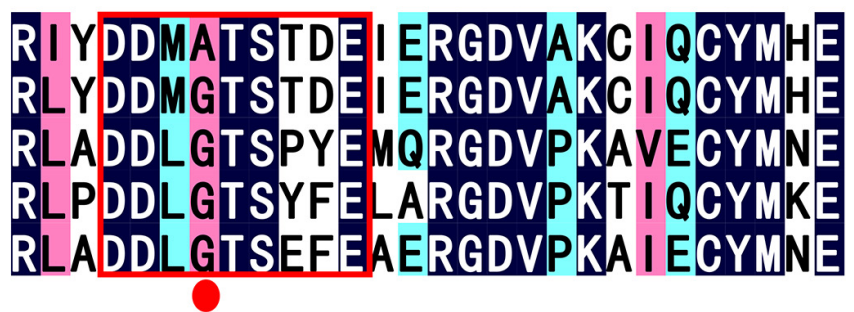

B

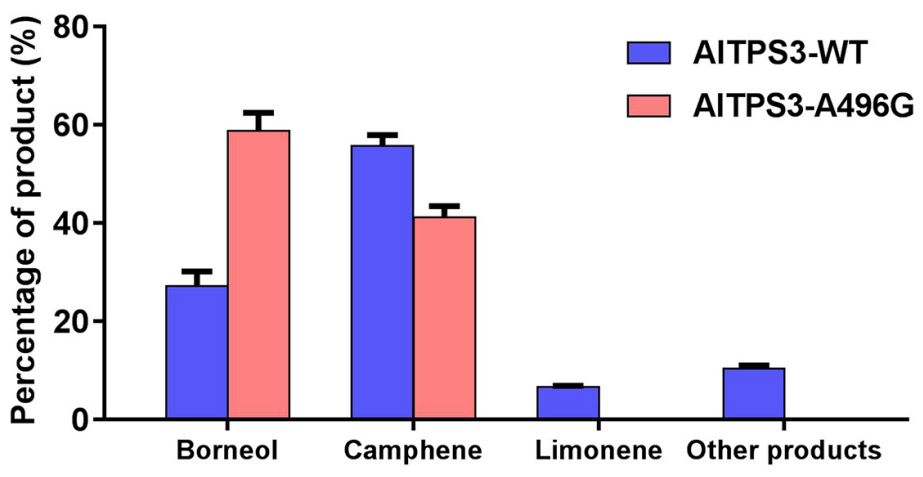

FIGURE 6 | The site-directed mutation of A496G in DTE motif of AITPS3 changed the major product from camphene to BPP. (A) Alignment of AITPS3 partial sequence with other BPPSs. The red box represents the DTE conserved motif. The red dot indicates the different amino acid, Ala vs. Gly, between AITPS3 and other BPPSs. (B) Product percentage of AITPS3-WT (wild type) and AITPS3-A496G.

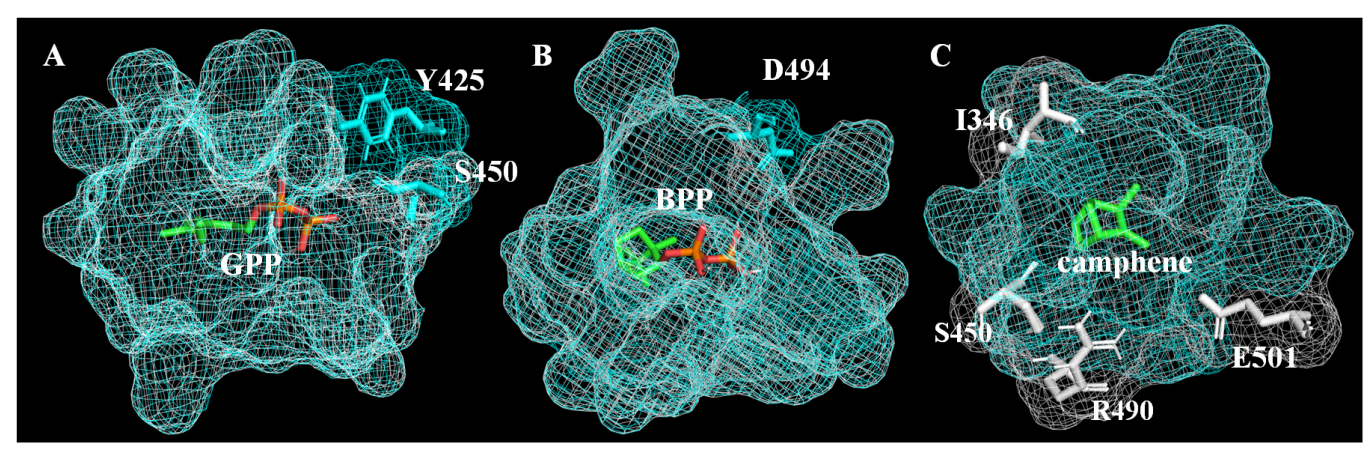

FIGURE 7 | Binding pockets of AITPS3-A496G/AITPS3-WT with substrate GPP (A), product BPP (B) and product camphene (C). Binding pockets of AITPS3-A496G (colored in blue) is superimposed on AITPS3-WT (colored in white). The main active and different amino acid residues are shown in sticks model, and the residues of AITPS3-A496G and AITPS3-WT are colored in blue and white, respectively. Substrate and products are colored in green and orange with the carbon atom shown in green and the phosphoric acid group shown in orange.

BPP as major product are glycine. The mutation of this special site of AlTPS3 (AlTPS3-A496G) changed the major product to BPP instead of camphene. A496 is located in the conserved motif of DTE, which is related to the binding of metal ions. Molecular docking suggests that A496G mutation narrows the camphenebinding pocket and decreases the BPP-binding energy, thus changes the major product from camphene to BPP. Therefore, an important amino acid site for product BPP selectivity was revealed in this study. The difference of AlTPS3 and AvBPPS in this site is one of the molecular bases for their enzymatic difference. Other sites in the catalytic pocket or active regions may also affect the BPP selectivity. More mutation experiments could be performed to validate the other active sites, but these experiments are beyond the scope of the present study and will be performed in a future work.

Besides of the enzymatic products difference, AlTPS3 and AvBPPS have expressional difference. Both AlTPS 3 and AvBPPS are highly expressed in 45-DAF seeds, but the expression level of $A v B P P S$ is significantly higher than that of AlTPS3, which is consistent with the total content of BPP-related terpenoids. Taken together, the higher BPP productivity and higher expression level of AvBPPS than those of AlTPS3 are the important part of molecular bases for difference of BPPrelated terpenoids, including bornyl acetate, between A. villosum 


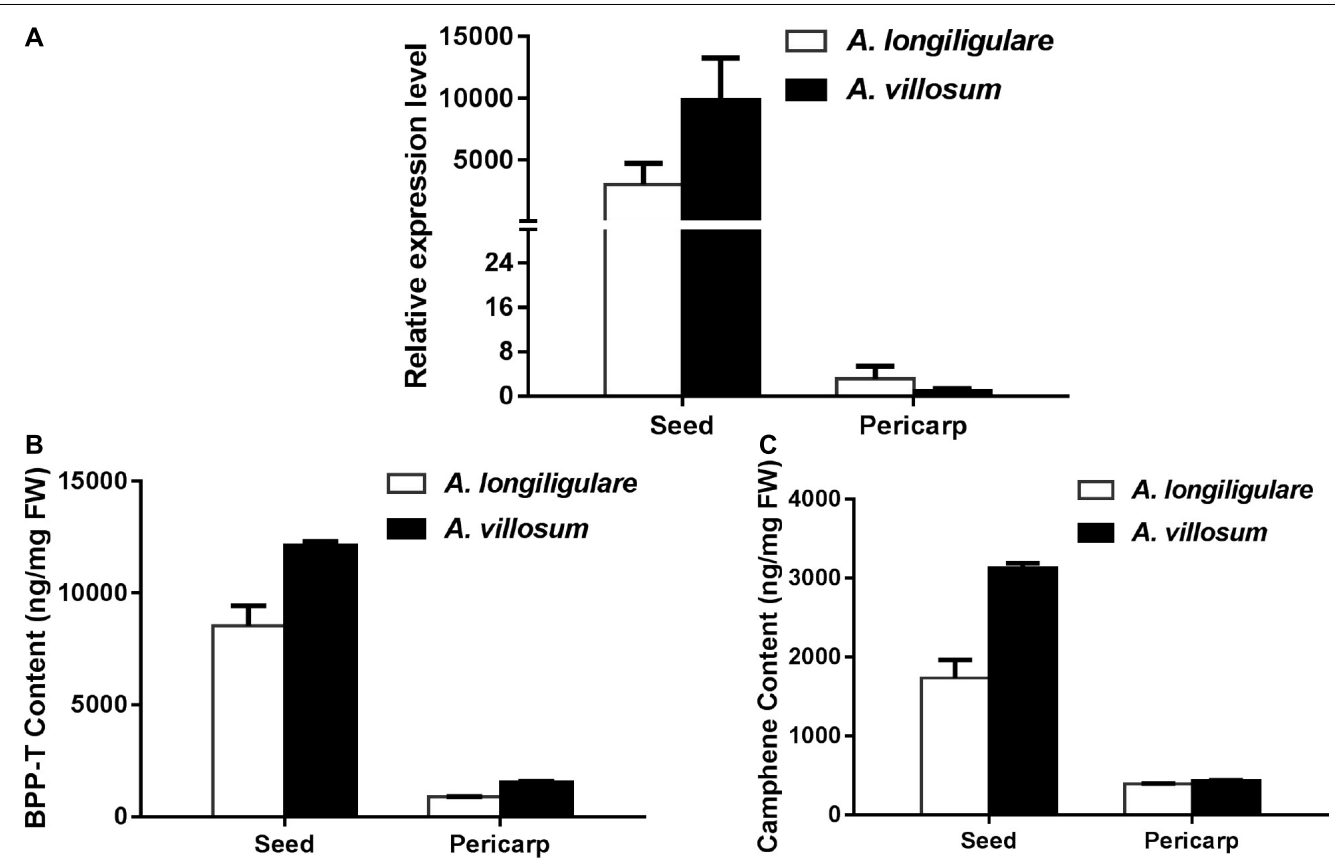

FIGURE 8 | Expression levels of AITPS3 and AvBPPS and related monoterpenoids in fruits of $A$. longiligulare and $A$. villosum. (A) The expression levels of AlTPS3 and AvBPPS. (B) The total content of BPP-related terpenoids. BPP-T means BPP-related terpenoids (borneol, camphor and bornyl acetate). (C) The content of camphene.

and A. longiligulare. According to Figure 1B, besides BPPS, the acyltransferase catalyzing borneol to form bornyl acetate and reductase catalyzing the reduction reaction to produce camphor from borneol are also critical for the bornyl acetate and camphor biosynthesis. Therefore, in order to further reveal the molecular bases for the bornyl acetate and camphor differences between these two species, our further study will mine the acyltransferase and reductase genes and compare their differences. Present study is the first step to explore the critical genes for the differential accumulation of bioactive terpenoids in these two species.

The previously study on the genetic identification for Fructus Amomi were mostly based on the non-coding sequences or genes unrelated to secondary metabolism. In this work, $B P P S$, the key gene involved in the biosynthesis of the active compounds was identified as a target gene that could be applied for the quality-related identification and breeding of Fructus Amomi. In addition, our study provides insight into the evolution of these two species on the functional genes involved in terpenoid biosynthesis, including the highly conserved genes as AvTPS 2 and AlTPS2, and the differential genes as AvBPPS and AlTPS3, which provides the foundation for the differentiation.

\section{DATA AVAILABILITY STATEMENT}

The datasets presented in this study can be found in online repositories. The names of the repository/repositories and accession number(s) can be found in the article/ Supplementary Material.

\section{AUTHOR CONTRIBUTIONS}

JY designed this study, analyzed the data, and wrote the manuscript. HZ, ML, YZ, XL, WW, HL, ZZ, and JW performed the experiments and analyzed the data. DM revised the manuscript. All authors contributed to the article and approved the submitted version.

\section{FUNDING}

This work was financially supported by the National Natural Science Foundation of China (81303163 and 81872954) and the Innovation Team Project of University of Guangdong Province (2016KYTD02).

\section{ACKNOWLEDGMENTS}

We thank Mr. Yushi Ye (South China Botanical Garden) and Guozhen He (Guangzhou University of Chinese Medicine) for collecting plant samples. We thank LetPub (www.letpub.com) for its linguistic assistance during the preparation of this manuscript.

\section{SUPPLEMENTARY MATERIAL}

The Supplementary Material for this article can be found online at: https://www.frontiersin.org/articles/10.3389/fpls.2021. 695551/full\#supplementary-material 


\section{REFERENCES}

Adam, K. P., and Croteau, R. (1998). Monoterpene Biosynthesis in the Liverwort Conocephalum Conicum: demonstration of Sabinene Synthase and Bornyl Diphosphate Synthase. Phytochemistry 49, 475-480. doi: 10.1016/S00319422(97)00741-3

Aprotosoaie, A. C., Hăncianu, M., Costache, I. I., and Miron, A. (2014). Linalool: a review on a key odorant molecule with valuable biological properties. Flavour Fragr. J. 29, 193-219. doi: 10.1002/ffj.3197

Chen, C., Zheng, Y., Zhong, Y., Wu, Y., Li, Z., Xu, L. A., et al. (2018). Transcriptome analysis and identification of genes related to terpenoid biosynthesis in Cinnamomum camphora. BMC Genomics 19:550. doi: 10.1186/s12864-0184941-1

Chen, N., Sun, G., Yuan, X., Hou, J., Wu, Q., Soromou, L. W., et al. (2014). Inhibition of lung inflammatory responses by bornyl acetate is correlated with regulation of myeloperoxidase activity. J. Surg. Res. 186, 436-445. doi: 10.1016/ j.jss.2013.09.003

Christianson, D. W. (2017). Structural and chemical biology of terpenoid cyclases. Chem. Rev. 117, 11570-11648. doi: 10.1021/acs.chemrev.7b00287

Commission of Chinese Materia Medica. (1999). Chinese Materia Medica, Vol. 8. Shanghai: Shanghai Science and Technology Publishing House.

Commission of Chinese Pharmacopoeia. (2015). Pharmacopoeia of the People's Republic of China. Beijing: China Medico-Pharmaceutical Science.

Cui, Y., Chen, X., Nie, L., Sun, W., Hu, H., Lin, Y., et al. (2019). Comparison and Phylogenetic Analysis of Chloroplast Genomes of Three Medicinal and Edible Amomum Species. Int. J. Mol. Sci. 20:4040. doi: 10.3390/ijms20164040

De, B. H. J., Vichith, L., and Lars, B. (2011). Steam sauna and mother roasting in Lao PDR: practices and chemical constituents of essential oils of plant species used in postpartum recovery. BMC Complement. Altern. Med. 11:128. doi: 10.1186/1472-6882-11-128

Despinasse, Y., Fiorucci, S., Antonczak, S., Moja, S., Bony, A., Nicole, F., et al. (2017). Bornyl-diphosphate synthase from Lavandula angustifolia: a major monoterpene synthase involved in essential oil quality. Phytochemistry 137, 24-33. doi: 10.1016/j.phytochem.2017.01.015

Doh, E. J., Kim, J. H., and Lee, G. (2019). Identification and Monitoring of Amomi Fructus and its Adulterants Based on DNA Barcoding Analysis and Designed DNA Markers. Molecules 24:4193. doi: 10.3390/molecules24224193

Finn, R. D., Coggill, P., Eberhardt, R. Y., Eddy, S. R., Mistry, J., Mitchell, A. L., et al. (2016). The Pfam protein families database: towards a more sustainable future. Nucleic Acids Res. 44, D279-D285. doi: 10.1093/nar/gkv1344

He, X., Wang, H., Yang, J., Deng, K., and Wang, T. (2018). RNA sequencing on Amomum villosum Lour. induced by MeJA identifies the genes of WRKY and terpene synthases involved in terpene biosynthesis. Genome 61, 91-102. doi: 10.1139/gen-2017-0142

Huang, Q., Duan, Z., Yang, J., Ma, X., Zhan, R., Xu, H., et al. (2014). SNP typing for germplasm identification of Amomum villosum Lour. Based on DNA barcoding markers. PLoS One 9:e114940. doi: 10.1371/journal.pone.0114940

Hurd, M. C., Kwon, M., and Ro, D. K. (2017). Functional identification of a Lippia dulcis bornyl diphosphate synthase that contains a duplicated, inhibitory arginine-rich motif. Biochem. Biophys. Res. Commun. 490, 963-968. doi: 10. 1016/j.bbrc.2017.06.147

Kim, S. H., Lee, S. Y., Hong, C. Y., Gwak, K. S., Park, M. J., Smith, D., et al. (2013). Whitening and antioxidant activities of bornyl acetate and nezukol fractionated from Cryptomeria japonica essential oil. Int. J. Cosmet. Sci. 35, 484-490. doi: 10.1111/ics.12069

Landmann, C., Fink, B., Festner, M., Dregus, M., Engel, K. H., and Schwab, W. (2007). Cloning and functional characterization of three terpene synthases from lavender (Lavandula angustifolia). Arch. Biochem. Biophys. 465, 417-429. doi: 10.1016/j.abb.2007.06.011

Li, J., and Wang, S. X. (2016). Synergistic enhancement of the antitumor activity of 5 -fluorouracil by bornyl acetate in SGC-7901 human gastric cancer cells and the determination of the underlying mechanism of action. J. BUON 21, 108-117.

Ma, R., Su, P., Guo, J., Jin, B., Ma, Q., Zhang, H., et al. (2021). Bornyl Diphosphate Synthase From Cinnamomum burmanni and Its Application for (+)-Borneol Biosynthesis in Yeast. Front. Bioeng. Biotechnol. 11:631863. doi: 10.3389/fbioe. 2021.631863

Magnard, J. L., Bony, A. R., Bettini, F., Campanaro, A., Blerot, B., Baudino, S., et al. (2018). Linalool and linalool nerolidol synthases in roses, several genes for little scent. Plant Physiol. Biochem. 127, 74-87. doi: 10.1016/j.plaphy.2018.03.009
Mei, J. D., Yuan, Z., Nan, Y. J., and Ming, X. X. (2015). Advances in research of pharmacological effects and formulation studies of linalool. China J. Chin. Materia 40, 3530-3533.

Qin, Q., Jiang, C. L., Jiang, M. L., Zhang, L. H., and Jiang, X. H. (2017). Compare on the Content of Bronyl Acetate and Total Volatile Oil in Fructus Amomi from Different Producing Area. Guid. J. Tradit. Chin. Med. Pharmacy 23, 70-72.

Tholl, D. (2015). Biosynthesis and Biological Functions of Terpenoids in Plants. Adv. Biochem. Eng. Biotechnol. 148, 63-106. doi: 10.1007/10_2014_295

Torrens-Spence, M. P., Pluskal, T., Li, F. S., Carballo, V., and Weng, J. K. (2018). Complete Pathway Elucidation and Heterologous Reconstitution of Rhodiola Salidroside Biosynthesis. Mol. Plant. 11, 205-217. doi: 10.1016/j.molp.2017.12. 007

Trott, O., and Olson, A. J. (2010). AutoDock Vina: improving the speed and accuracy of docking with a new scoring function, efficient optimization and multithreading. J. Computat. Chem. 31, 455-461. doi: 10.1002/jcc.21334

Wang, H., Ma, D., Yang, J., Deng, K., Li, M., Ji, X., et al. (2018). An Integrative Volatile Terpenoid Profiling and Transcriptomics Analysis for Gene Mining and Functional Characterization of AvBPPS and AvPS Involved in the Monoterpenoid Biosynthesis in Amomum villosum. Front. Plant Sci. 9:846. doi: 10.3389/fpls.2018.00846

Wang, H., Wei, J., Yang, J., Zhan, R., Chen, W., and Yuan, Y. (2014). Overexpression of HMGR and DXR from Amomum villosum Lour. Affects the Biosynthesis of Terpenoids in Tobacco. World Sci. Technol. 7, 1513-1527.

Wei, G., Tian, P., Zhang, F., Qin, H., Miao, H., Chen, Q., et al. (2016). Integrative Analyses of Nontargeted Volatile Profiling and Transcriptome Data Provide Molecular Insight into VOC Diversity in Cucumber Plants (Cucumis sativus). Plant Physiol. 172, 603-618. doi: 10.1104/pp.16.01051

Whittington, D. A., Wise, M. L., Urbansky, M., Coates, R. M., Croteau, R. B., and Christianson, D. W. (2002). Bornyl diphosphate synthase: structure and strategy for carbocation manipulation by a terpenoid cyclase. Proc. Natl. Acad. Sci. U. S. A. 99, 15375-15380. doi: 10.1073/pnas.232591099

Wise, M. L., Savage, T. J., Katahira, E., and Croteau, R. (1998). Monoterpene synthases from common sage (Salvia officinalis). cDNA isolation, characterization, and functional expression of (+)-sabinene synthase, 1,8cineole synthase, and (+)-bornyl diphosphate synthase. J. Biol. Chem. 273, 14891-14899. doi: 10.1074/jbc.273.24.14891

Xue, X., Yang, D., Wang, D., Xu, X., Zhu, L., and Zhao, Z. (2015). Solidification of floating organic drop liquid-phase microextraction cell fishing with gas chromatography-mass spectrometry for screening bioactive components from Amomum villosum Lour. Biomed. Chromatogr. 29, 626-632. doi: 10.1002/bmc. 3324

Yang, H., Zhao, R., Chen, H., Jia, P., Bao, L., and Tang, H. (2014). Bornyl acetate has an anti-inflammatory effect in human chondrocytes via induction of IL-11. IUBMB Life 66, 854-859. doi: 10.1002/iub.1338

Yang, J., Adhikari, M. N., Liu, H., Xu, H., He, G., Zhan, R., et al. (2012). Characterization and functional analysis of the genes encoding 1-deoxy-Dxylulose-5-phosphate reductoisomerase and 1-deoxy-D-xylulose-5-phosphate synthase, the two enzymes in the MEP pathway, from Amomum villosum Lour. Mol. Biol. Rep. 39, 8287-8296. doi: 10.1007/s11033-012-1676-y

Yu, J. G., Luo, X. Z., Lan, S., Li, D. Y., and Liu, C. Y. (2005). Chemical constituents from seeds of Annona squamosa. Acta Pharm. Sin. 40, 153-158.

Conflict of Interest: The authors declare that the research was conducted in the absence of any commercial or financial relationships that could be construed as a potential conflict of interest.

Publisher's Note: All claims expressed in this article are solely those of the authors and do not necessarily represent those of their affiliated organizations, or those of the publisher, the editors and the reviewers. Any product that may be evaluated in this article, or claim that may be made by its manufacturer, is not guaranteed or endorsed by the publisher.

Copyright (C) 2021 Zhao, Li, Zhao, Lin, Liang, Wei, Wei, Ma, Zhou and Yang. This is an open-access article distributed under the terms of the Creative Commons Attribution License (CC BY). The use, distribution or reproduction in other forums is permitted, provided the original author(s) and the copyright owner(s) are credited and that the original publication in this journal is cited, in accordance with accepted academic practice. No use, distribution or reproduction is permitted which does not comply with these terms. 